\title{
RECHERCHE À VOISINAGE VARIABLE DE GRAPHES EXTRÉMAUX 26. NOUVEAUX RÉSULTATS SUR LA MAILLE
}

\author{
Mustapha Aouchiche ${ }^{1}$, Odile Favaron $^{2,3}$ \\ ET Pierre Hansen ${ }^{4}$
}

Résumé. On étudie à l'aide du système AutoGraphiX 2 (AGX 2) des relations de la forme

$$
\underline{b}_{n} \leq g \oplus i \leq \bar{b}_{n}
$$

où $g$ désigne la maille d'un graphe $G=(V, E), i$ un autre invariant parmi la distance moyenne $\bar{l}$, l'index $\lambda_{1}$, l'indice de Randić $R$ et le nombre de domination $\beta, \oplus$ désigne l'une des opérations,,$+- \times, /$, $\underline{b}_{n}$ et $\bar{b}_{n}$ des fonctions de l'ordre $n$ du graphe qui bornent l'expression $g \oplus i$ et sont atteintes pour tout $n$ (sauf éventuellement de très petites valeurs du fait des effets de bord). Les résultats prouvés ou discutés ci-dessous ont déjà été présentés, sous forme de conjectures, dans un article précédent paru dans RAIRO Recherche Opérationnelle [RAIRO Oper. Res. 39 (2005) 275-293].

Mots Clés. Graphe, AGX, maille, distance, Randić, index, domination.

Reçu le 4 juin, 2008. Accepté le 7 mars, 2009.

1 HEC Montréal, Qc, Canada; Mustapha.Aouchiche@gerad.ca

2 Univ Paris-Sud, LRI, UMR 8623, Orsay, 91405, France.

3 CNRS, Orsay, 91405, France; of@LRI.lri.fr

${ }^{4}$ GERAD et HEC Montréal, Qc, Canada; Pierre.Hansen@gerad.ca 


\begin{abstract}
Using the AutoGraphiX 2 system (AGX2), we study relations between graph invariants of the form

$$
\underline{b}_{n} \leq g \oplus i \leq \bar{b}_{n}
$$

where $g$ denotes the girth of a graph $G=(V, E), i$ another invariant among the average distance $\bar{l}$, the index $\lambda_{1}$, the Randić index $R$ and the domination number $\beta, \oplus$ denotes one of the four operations,,$+- \times, /$, $\underline{b}_{n}$ and $\bar{b}_{n}$ lower and upper bounding functions of the order $n$ of the graph considered which are tight for all $n$ (except possibly very small values due to border effects). The results proved or discussed below were first presented as conjectures in a previous paper published in RAIRO Operations Research [RAIRO Oper. Res. 39 (2005) 275-293].
\end{abstract}

Keywords. Graph, AGX, girth, distance, Randić, index, domination.

Classification Mathématique. 05C35, 05C12.

\title{
1. INTRODUCTION
}

Soit $G=(V, E)$ un graphe simple et connexe, où $V$ est l'ensemble de ses sommets et $E$ l'ensemble de ses arêtes. La longueur d'un cycle est le nombre de ses arêtes (ou sommets). La maille d'un graphe $G$, notée $g$, est la longueur du plus petit cycle de $G$. La distance $d(u, v)$ entre deux sommets $u$ et $v$ de $G$ est la longueur du plus court chemin les reliant. La distance moyenne d'un graphe $G$, notée $\bar{l}(G)$, ou simplement $\bar{l}$ s'il n'y a pas de risque de confusion, est la moyenne des distances entre les paires de sommets de $G$. La matrice d'adjacence $A$ d'un graphe $G$ est la matrice dont les éléments sont définis par $a_{i j}=1$ si les sommets $v_{i}$ et $v_{j}$ sont voisins dans $G$ et $a_{i j}=0$ sinon. L'index $\lambda_{1}$ de $G$ est la plus grande valeur propre de la matrice d'adjacence $A$ de $G$. Pour un sommet $v_{i}$ de $G, d_{i}=d\left(v_{i}\right)$ est le degré de $v_{i}, c-\grave{a}-d$, le nombre de ses voisins. L'indice de Randić d'un graphe $G=(V, E)$ est défini par

$$
R=\sum_{v_{i} v_{j} \in E} \frac{1}{\sqrt{d_{i} d_{j}}} .
$$

Un sous-ensemble de sommets $W$ de $G$ est un ensemble dominant si tout sommet de $G-W$ possède un voisin dans $W$. Le nombre de domination $\beta$ de $G$ est la cardinalité du plus petit ensemble dominant de $G$.

Dans cet article, nous nous intéressons aux résultats de la forme suivante, dite Forme 1 d'AGX $[1,2]$

$$
\underline{b}_{n} \leq i_{1} \oplus i_{2} \leq \bar{b}_{n}
$$


où $i_{1}$ et $i_{2}$ sont deux invariants graphiques, $\oplus$ désigne l'une des opérations ,,$+- \times, /, \underline{b}_{n}$ et $\bar{b}_{n}$ des fonctions de l'ordre $n$ du graphe qui bornent l'expression $i_{1} \oplus i_{2}$ et sont serrées pour tout $n$, sauf éventuellement pour de très petites valeurs du fait des effets de bord. Plus particulièrement notre intérêt est concentré sur le cas où $i_{1}$ représente la maille $g$ d'un graphe, $c$-à- $d$, les résultats de la forme

$$
\underline{b}_{n} \leq g \oplus i \leq \bar{b}_{n}
$$

où $i$ est un autre invariant parmi la distance moyenne $\bar{l}$, l'index $\lambda_{1}$, l'indice de Randić $R$ et le nombre de domination $\beta$.

Cet article fait suite à [4]. Dans ce dernier, des résultats de la forme (1.2) ont été démontrés, où $i$ était le nombre de stabilité $\alpha$, le rayon $r$, le diamètre $D$, les degrés maximum $\Delta$, moyen $\bar{d}$ et minimum $\delta$. Dans ce même article 12 conjectures ont été données dont une, à savoir $\chi \cdot g \leq 3 n$, a été démontrée par un rapporteur anonyme de l'article et une autre, soit $\lambda_{1} \cdot g \leq 3(n-1)$, réfutée par ce même rapporteur. Deux des dix conjectures restantes, relatives à l'indice de Randić ont été prouvées dans [5]. Sept des huit autres conjectures sont démontrées ci-dessous. Nous donnons aussi des contre-exemples ainsi qu'une reformulation et des résultats partiels relatifs à la dernière conjecture, à savoir la borne inférieure incorrecte sur $\bar{l} / g$.

Toutes les bornes présentées dans [4] et prouvées dans [4] ou ci-dessous ont été obtenues en premier lieu comme des conjectures par le système AGX $2[3,8]$, qui permet entre autres fonctions la génération de conjectures en théorie des graphes. Comme nous l'avons déjà mentionné, les résultats de la forme (1.2) sont des cas particuliers de résultats de la forme (1.1). Les très nombreux résultats de forme (1.1) obtenus lors de la comparaison systématique de 20 invariants sont donnés en détails dans [1] et résumés dans [2]. Les preuves complètes (ou, rarement, les réfutations) de ces conjectures sont présentées dans une série d'articles, à laquelle appartient également celui-ci, sous le titre commun Variable Neighborhood Search for Extremal Graphs.

Dans la suite de cet article, les résultats faisant intervenir, outre la maille, la distance moyenne $\bar{l}$ sont discutés dans la Section 2; ceux concernant l'index $\lambda_{1}$ sont présentés dans la Section 3; deux bornes impliquant l'indice de Randić $R$ sont prouvées dans la Section 4; et enfin la Section 5 est consacrée au nombre de domination $\beta$.

\section{LA DISTANCE MOYENNE}

On appelle monocle $S_{p, q}[7]$ un graphe obtenu à partir d'un cycle $C_{p}$ sur $p \geq 3$ sommets et un chemin $P_{q}$ sur $q \geq 0$ sommets en ajoutant une arête reliant une des extrémités de $P_{q}$ à un sommet de $C_{p}$. Si $q=0$, le monocle $S_{p, 0}$ est le cycle $C_{p}$. Par définition, $S_{p, q}$ contient $p+q$ sommets et $p+q$ arêtes. 
Quelques lemmes sont requis pour le résultat principal de cette section.. La transmission $\sigma_{G}(u)$ d'un sommet d'un graphe $G$ est la somme des distances de $u$ à tous les autres sommets de $G$. La transmission $\sigma(G)$ est la moitié de la somme des transmissions de tous les sommets de $G$, ou encore la somme des distances entre toutes les paires de sommets de $G$. D'où, la distance moyenne de $G$ est

$$
\bar{l}=\frac{2 \sigma(G)}{n(n-1)}
$$

Lemme 2.1. Si un graphe $G$ est constitué de deux graphes $G_{1}$ et $G_{2}$ ayant exactement un sommet commun u, alors

$$
\sigma(G)=\sigma\left(G_{1}\right)+\sigma\left(G_{2}\right)+\sigma_{G_{1}}(u) \cdot\left(n_{2}-1\right)+\sigma_{G_{2}}(u) \cdot\left(n_{1}-1\right)
$$

où $n_{1}$ et $n_{2}$ sont les nombres de sommets de $G_{1}$ et $G_{2}$ respectivement.

Démonstration. Soit $V\left(G_{i}\right)=V_{i}$ pour $i=1,2$. Alors

$$
\begin{aligned}
\sigma(G) & =\sigma\left(G_{1}\right)+\sigma\left(G_{2}\right)+\sum_{x \in V_{1} \backslash\{u\}, y \in V_{2} \backslash\{u\}}(d(x, u)+d(y, u)) \\
& =\sigma\left(G_{1}\right)+\sigma\left(G_{2}\right)+\sum_{y \in V_{2} \backslash\{u\}} \sum_{x \in V_{1} \backslash\{u\}}(d(x, u)+d(y, u)) \\
& =\sigma\left(G_{1}\right)+\sigma\left(G_{2}\right)+\sum_{y \in V_{2} \backslash\{u\}}\left(\sigma_{G_{1}}(u)+\left(n_{1}-1\right) d(y, u)\right) \\
& =\sigma\left(G_{1}\right)+\sigma\left(G_{2}\right)+\sigma_{G_{1}}(u) \cdot\left(n_{2}-1\right)+\sigma_{G_{2}}(u) \cdot\left(n_{1}-1\right) .
\end{aligned}
$$

Ce qui prouve le lemme.

Lemme 2.2. La somme des distances $\sigma$ pour un monocle $S_{p, n-p}$ est donnée par

$$
2 \sigma\left(S_{p, n-p}\right)=\left\{\begin{array}{cl}
\frac{5}{12} p^{3}-\frac{n+2}{2} p^{2}+\left(n+\frac{7}{12}\right) p+\frac{n^{3}}{3}-\frac{5 n}{6} & \text { si p est impair } \\
\frac{5}{12} p^{3}-\frac{n+2}{2} p^{2}+\left(n+\frac{1}{3}\right) p+\frac{n^{3}-n}{3} & \text { si } p \text { est pair. }
\end{array}\right.
$$


Démonstration. On applique le Lemme 2.1 avec $G_{1}=C_{p}$ et $G_{2}=P_{n-p+1}$. Si $p$ est impair,

$$
\begin{aligned}
2 \sigma\left(S_{p, n-p}\right)= & \frac{p\left(p^{2}-1\right)}{4}+\frac{(n-p)(n-p+1)(n-p+2)}{3} \\
& +2\left((p-1) \frac{(n-p)(n-p+1)}{2}+(n-p) \frac{p^{2}-1}{4}\right) \\
= & \frac{p^{2}-1}{4}(2 n-p)+\frac{(n-p)(n-p+1)}{3}(n+2 p-1) \\
= & \frac{5}{12} p^{3}-\frac{n+2}{2} p^{2}+\left(n+\frac{7}{12}\right) p+\frac{n^{3}}{3}-\frac{5 n}{6} .
\end{aligned}
$$

Si $p$ est pair,

$$
\begin{aligned}
2 \sigma\left(S_{p, n-p}\right)= & \frac{p^{3}}{4}+\frac{(n-p)(n-p+1)(n-p+2)}{3} \\
& +2\left((p-1) \frac{(n-p)(n-p+1)}{2}+(n-p) \frac{p^{2}}{4}\right) \\
= & \frac{p^{3}}{4}-\frac{p^{3}}{2}+\frac{n p^{2}}{2}+(n-p)(n-p+1)\left(\frac{n-p+2}{3}+p-1\right) \\
= & \frac{5}{12} p^{3}-\frac{n+2}{2} p^{2}+\left(n+\frac{1}{3}\right) p+\frac{n^{3}-n}{3} .
\end{aligned}
$$

Ce qui complète la preuve.

Lemme 2.3. Les fonctions $f:[3, n] \longrightarrow R$ et $h:[3, n] \longrightarrow R$ définies par

$$
\begin{aligned}
& f(t)=\frac{5 t^{4}}{12}-\frac{n+2}{2} t^{3}+\left(n+\frac{7}{12}\right) t^{2}+\left(\frac{n^{3}}{3}-\frac{5 n}{6}\right) t \\
& h(t)=\frac{5 t^{4}}{12}-\frac{n+2}{2} t^{3}+\left(n+\frac{1}{3}\right) t^{2}+\left(\frac{n^{3}}{3}-\frac{n}{3}\right) t
\end{aligned}
$$

sont croissantes.

Démonstration. La dérivée

$$
f^{\prime}(t)=\frac{5 t^{3}}{3}-3 \frac{n+2}{2} t^{2}+\left(2 n+\frac{7}{6}\right) t+\left(\frac{n^{3}}{3}-\frac{5 n}{6}\right)
$$

de $f(t)$ est un polynôme de degré 3 . Le discriminant (calculé à l'aide de Maple) $4 P^{3}+27 Q^{2}$ de sa forme réduite $t^{3}+P t+Q$ est

$$
\text { Disc }=\frac{993}{250} n^{6}-\frac{81}{125} n^{5}-\frac{41751}{2500} n^{4}+\frac{1296}{625} n^{3}+\frac{159543}{10000} n^{2}-\frac{747}{2500} n-\frac{539}{2500} .
$$


Pour $n \geq 3$,

$$
\text { Disc } \geq n^{4} \cdot\left(\frac{993}{250} n^{2}-\frac{81}{125} n-\frac{41751}{2500}\right)>0 .
$$

Alors, $f^{\prime}$ a une seule racine réelle qui est négative, donc $f^{\prime}(0)>0$. Par conséquent $f^{\prime}(t)$ est positive, et $f$ est croissante sur $[3, n]$.

Pour la deuxième fonction, il est facile de voir que

$$
h(t)=f(t)+\left(\frac{n t}{2}-\frac{t^{2}}{4}\right) ;
$$

donc $h$ est somme de deux fonctions croissantes sur $[3, n]$.

Théorème 2.1 (Conjecture 2 de [4]). Pour tout graphe connexe $G$ d'ordre $n \geq 3$, de maille finie $g$ et de distance moyenne $\bar{l}$,

$$
\bar{l} g \leq \begin{cases}\frac{n^{3}}{4(n-1)} & \text { si } n \text { est pair, } \\ \frac{n^{2}+n}{4} & \text { si } n \text { est impair }\end{cases}
$$

avec égalité si et seulement si $G$ est un cycle.

Démonstration L'ordre $n$ étant fixé, considérons un graphe $G$ tel que $\bar{l}(G) \cdot g(G)$ soit maximum, ou de façon équivalente, $\sigma(G) \cdot g(G)$ soit maximum.

Lemme 2.4. $G$ est unicyclique.

Démonstration du Lemme 2.4. Si $G$ n'est pas unicyclique, soit $C$ un cycle de $G$ sur $g$ sommets et $G^{\prime}$ un graphe partiel couvrant de $G$ obtenu à partir de $G$ par la suppression d'une arête de chaque cycle de $G$ autre que $C$. Alors $g\left(G^{\prime}\right)=g$ et $\sigma\left(G^{\prime}\right)>\sigma(G)$, ce qui est en contradiction avec le choix de $G$.

Lemme 2.5. $\Delta(G) \leq 3$ et tous les sommets de degré 3, s'ils existent, appartiennent à l'unique cycle de $G$.

Démonstration du Lemme 2.5. Si le Lemme 2.5 était faux, alors $G$ contiendrait un sommet $x$ de degré au moins 3 et deux chemins pendants $x y_{1} y_{2} \ldots y_{r}$ et $x z_{1} z_{2} \ldots z_{s}$ qui seraient disjoints de $C$, sauf éventuellement en $x$. Pour ces deux chemins $d\left(y_{r}\right)=d\left(z_{s}\right)=1$ et $d\left(y_{i}\right)=d\left(z_{j}\right)=2$ pour $1 \leq i<r$ et $1 \leq j<s$. Supposons, sans perte de généralité, que $1 \leq s \leq r$ et soit $V^{\prime}=\left\{x, y_{1}, \ldots y_{r}, z_{1}, \ldots z_{s}\right\}$. Le graphe $G^{\prime}=\left(G-z_{s-1} z_{s}\right) \cup y_{r} z_{s}$ (avec la convention $z_{0}=x$ ) a la même maille que $G$. De plus

$$
\begin{aligned}
\sum_{u, v \in V^{\prime}} d_{G^{\prime}}(u, v) & =\sum_{u, v \in V^{\prime}} d_{G}(u, v)=\sigma\left(P_{r+s+1}\right), \\
\sum_{u \in V \backslash\left\{z_{s}\right\}, v \in V \backslash V^{\prime}} d_{G^{\prime}}(u, v) & =\sum_{u \in V \backslash\left\{z_{s}\right\}, v \in V \backslash V^{\prime}} d_{G}(u, v), \\
d_{G^{\prime}}\left(z_{s}, v\right) & >d_{G}\left(z_{s}, v\right) \text { pour tout } v \in V \backslash V^{\prime} . .
\end{aligned}
$$

Par conséquent, $\sigma\left(G^{\prime}\right)>\sigma(G)$, ce qui est en contradiction avec le choix de $G$. 
Lemme 2.6. G est un monocle.

Preuve du Lemme 2.6. D'après le Lemme 2.5, $G$ est constitué d'un cycle $C$ et d'au plus un chemin pendant relié à chaque sommet de $C$. Si $G$ n'est pas un monocle, soit $y_{0} y_{1} y_{2} \ldots y_{r}$ et $z_{0} z_{1} z_{2} \ldots z_{s}$ deux chemins de $G$ avec $y_{0}$ et $z_{0}$ dans $C$ et tels que $d\left(y_{0}, z_{0}\right)$ soit minimum. Soit $y_{0} a_{1} a_{2} \ldots a_{t} z_{0}$ un plus court chemin reliant $y_{0}$ et $z_{0}$ dans $C$, et soit $V^{\prime}=\left\{y_{0}, y_{1}, \ldots y_{r}, z_{0}, z_{1}, \ldots z_{s}, a_{1}, \ldots a_{t}\right\}$. Par la minimalité de $d\left(y_{0}, z_{0}\right)$, on a $d\left(a_{i}\right)=2$ pour tout $1 \leq i \leq t$, et $V \backslash V^{\prime} \neq \phi$. Comme $\left|V \backslash V^{\prime}\right| \neq 0$, au moins une des deux inégalités

$$
\begin{gathered}
\sum_{v \in V \backslash V^{\prime}} d_{G}\left(z_{s}, v\right)+\left|V \backslash V^{\prime}\right|>\sum_{v \in V \backslash V^{\prime}} d_{G}\left(y_{r}, v\right), \\
\sum_{v \in V \backslash V^{\prime}} d_{G}\left(y_{r}, v\right)+\left|V \backslash V^{\prime}\right|>\sum_{v \in V \backslash V^{\prime}} d_{G}\left(z_{s}, v\right)
\end{gathered}
$$

est satisfaite. Supposons, sans perte de généralité, que la première soit vraie. Le graphe $G^{\prime}=\left(G-y_{r} y_{r-1}\right) \cup\left\{y_{r} z_{s}\right\}$ a la même maille que $G$. Pour l'ensemble de sommets $V^{\prime}$, on a

$$
\sum_{u, v \in V^{\prime}} d_{G^{\prime}}(u, v)=\sum_{u, v \in V^{\prime}} d_{G}(u, v)=\sigma\left(P_{r+s+t+2}\right) .
$$

Si $u \in V \backslash\left\{y_{r}\right\}$ et $v \in V \backslash V^{\prime}$, alors $d_{G^{\prime}}(u, v)=d_{G}(u, v)$. Enfin, pour tout $v \in V \backslash V^{\prime}$, $d_{G^{\prime}}\left(y_{r}, v\right)=d_{G}\left(z_{s}, v\right)+1$ qui implique

$$
\begin{aligned}
\sum_{v \in V \backslash V^{\prime}} d_{G^{\prime}}\left(y_{r}, v\right) & =\sum_{v \in V \backslash V^{\prime}}\left(d_{G}\left(z_{s}, v\right)+1\right) \\
& =\sum_{v \in V \backslash V^{\prime}} d_{G}\left(z_{s}, v\right)+\left|V \backslash V^{\prime}\right|>\sum_{v \in V \backslash V^{\prime}} d_{G}\left(y_{r}, v\right) .
\end{aligned}
$$

Par conséquent, $\sigma\left(G^{\prime}\right)>\sigma(G)$, ce qui est en contradiction avec le choix de $G$. Donc $G$ doit être un monocle éventuellement réduit à un cycle si $g=n$.

Revenons maintenant à la preuve du théorème. D'après le Lemme 2.6, $G$ est un monocle $S_{g, n-g}$ avec $3 \leq g \leq n$. D'après le Lemme 2.6, $2 \sigma(G) \cdot g(G)$ est égal à $f(g)$ si $g$ est impair et à $h(g)$ si $g$ est pair, où $f$ et $h$ sont les fonctions croissantes étudiées dans le Lemme 2.3. Comme $g \leq n$, le maximum de $2 \sigma(G) \cdot g(G)$ est atteint pour $f(n)$ ou $h(n-1)$ si $n$ est impair, pour $f(n-1)$ ou $h(n)$ si $n$ est pair. Il est facile de voir que

$$
\begin{aligned}
& f(n)-h(n-1)=\frac{n^{3}}{2}-\frac{9 n^{2}}{4}+\frac{7 n}{2}-\frac{7}{4}>0, \\
& h(n)-f(n-1)=\frac{n^{3}}{2}-\frac{7 n^{2}}{4}+\frac{7 n}{2}-2>0 .
\end{aligned}
$$

Alors, $\bar{l} g$ est maximum pour un cycle. 
Un navet, noté $T_{n, g}$, est un graphe sur $n$ sommets composé d'un cycle sur $g$ sommets et $n-g$ arêtes pendantes, toutes incidentes au même sommet du cycle.

Conjecture 2.1 [4]. Soit $G=(V, E)$ un graphe connexe sur $n \geq 3$ sommets avec une maille finie $g$ et une distance moyenne $\bar{l}$. Alors

$$
\overline{\bar{l}} \geq\left\{\begin{array}{cl}
\frac{n}{4(n-1)} & \text { si } n \text { est pair } \\
\frac{n+1}{4 n} & \text { si } n \text { est impair }
\end{array}\right.
$$

avec égalité si et seulement si $G$ est le cycle $C_{n}$.

Cette conjecture est fausse. Le plus petit contre-exemple connu est $T_{31,15}$, pour lequel $\bar{l} / g \simeq 0.25749$, et la valeur correspondante pour un cycle $C_{31}$ est $\frac{8}{31} \simeq 0.25806$. En fait, pour $n \geq 31$ le cycle n'est pas le graphe extrême pour la borne inférieure sur $\bar{l} / g$. Alors, on a la nouvelle conjecture ci-dessous.

Conjecture 2.2. Soit $G=(V, E)$ un graphe connexe sur $n \geq 3$ sommets avec une maille finie $g$ et une distance moyenne $\bar{l}$.

(i) Si $n \leq 30$, alors

$$
\frac{\bar{l}}{g} \geq\left\{\begin{array}{cl}
\frac{n}{4(n-1)} & \text { si } n \text { est pair } \\
\frac{n+1}{4 n} & \text { si } n \text { est impair }
\end{array}\right.
$$

avec égalité si et seulement si $G$ est le cycle $C_{n}$.

(ii) Si $n \geq 31$, alors $\bar{l} / g$ est minimum pour un navet $T_{n, g}$.

Notons que si $n \geq 35$ la valeur de la borne inférieure (obtenue par AGX) est inférieure à $1 / 4$, tandis que pour un cycle la valeur correspondante est supérieure à $1 / 4$.

Les graphes extrêmes obtenus avec AGX pour $n \geq 31$ sont tous des navets, toutefois les paramètres définissant ces graphes n'ont pas assez de régularités apparentes pour pouvoir extraire une formule algébrique correspondant à la borne. Le tableau ci-dessous donne les valeurs de leur maille pour $31 \leq n \leq 190$.

\begin{tabular}{|c|c|c|c|c|c|c|c|c|}
\hline$n$ & {$[31,38]$} & {$[39,56]$} & {$[57,74]$} & {$[75,93]$} & {$[94,115]$} & {$[116,138]$} & {$[139,163]$} & {$[164,190]$} \\
\hline$g$ & 15 & 17 & 19 & 21 & 23 & 25 & 27 & 29 \\
\hline
\end{tabular}

Si AGX 2 fait une erreur dans ce cas, c'est dû au fait que seuls les graphes d'ordre $n \leq 20$ ont été étudié et que le plus petit contre-exemple connu a $n=31$ sommets. Une nouvelle fonction d'AGX 2, en cours de développement, permettra de tester les 
conjectures obtenues sur des graphes virtuels appartenant aux principales familles de graphes extrêmes rencontrées. Dans le cas présent, une telle fonction aurait permis de réfuter la conjecture 1 de [4] en calculant $\bar{l} / g$ en fonction de $n$ pour de grandes valeur de $n$ à l'aide de formules donnant $\bar{l}$ pour de grandes valeurs de $n$ et $g$ pour la famille des navets.

Nous donnons maintenant quelques résultats reliés à cette conjecture.

Proposition 2.1. Si $g=3$, alors $\bar{l} / g \geq 1 / 3$ avec égalité si et seulement si $G$ est le graphe complet $K_{n}$.

Proposition 2.2. Sig $=4$, alors

$$
\frac{\bar{l}}{g} \geq\left\{\begin{array}{cl}
\frac{3}{8}-\frac{1}{8(n-1)} & \text { si } n \text { est pair, } \\
\frac{3}{8}-\frac{1}{8 n} & \text { si } n \text { est impair },
\end{array}\right.
$$

avec égalité si et seulement si $G$ est le graphe biparti complet équilibré $K_{\left\lceil\frac{n}{2}\right\rceil,\left\lfloor\frac{n}{2}\right\rfloor}$.

Démonstration. Soit $G=(V, E)$ un graphe sur $n \geq 4$ sommets de taille $m$ et de maille $g=4$. Il est facile de voir que

$$
\bar{l} \geq 2-\frac{2 m}{n(n-1)} .
$$

Donc $\bar{l}$ est minimum si et seulement si $m$ est maximum (sous la condition $g=4$ ). Dans ce cas, en utilisant le théorème de Turan [17] (le nombre maximum d'arêtes dans un graphe sans $\left.K_{3}\right), m \leq\lfloor n / 2\rfloor \cdot\lceil n / 2\rceil$ avec égalité si et seulement si $G$ est le graphe biparti complet équilibré $K_{\left\lceil\frac{n}{2}\right\rceil,\left\lfloor\frac{n}{2}\right\rfloor}$.

Proposition 2.3. Si $G$ est un graphe unicyclique de maille fixée $g \leq n-1$, alors

$$
\bar{l} \geq\left\{\begin{array}{cl}
\frac{2}{g}-\frac{g^{2}-2 n g+8(n-1)}{4 n(n-1)} & \text { si g est pair, } \\
\frac{2}{g}-\frac{g^{3}-2 n g^{2}+(8 n-9) g+2 n}{4 g n(n-1)} & \text { si g est impair },
\end{array}\right.
$$

avec égalité si et seulement si $G$ est le navet $T_{n, g}$.

Démonstration. Soit $G$ un graphe unicyclique de maille $g \leq n-1$. Soit $u$ un sommet de degré au moins 3 sur l'unique cycle $C$ de $G$ et notons $u_{1}$ et $u_{2}$ les voisins de $u$ sur $C$. La suppression des deux arêtes $u u_{1}$ et $u u_{2}$ sépare $G$ en deux composantes connexes. Le sous-graphe induit par les sommets de la composante contenant $u$ est appelée branche de $G$ enracinée en $u$. Soit $V_{1}$ l'ensemble des sommets de cette branche et $V_{2}=\left(V \backslash V_{1}\right) \cup\{u\}$. Notons $G_{1}$ et $G_{2}$ les sous-graphes de $G$ induits par $V_{1}$ et $V_{2}$ respectivement et posons $n_{1}=\left|V_{1}\right|$ et $n_{2}=\left|V_{2}\right|$. En utilisant le Lemme 2.1, on obtient

$$
\sigma(G)=\sigma\left(G_{1}\right)+\sigma\left(G_{2}\right)+\left(n_{1}-1\right) \sigma_{G_{2}}(u)+\left(n_{2}-1\right) \sigma_{G_{1}}(u) .
$$


Notons que $G_{1}$ est un arbre. Donc

$$
\sigma\left(G_{1}\right) \geq \sigma\left(S_{n_{1}}\right) \quad \text { et } \quad \sigma_{G_{1}}(u) \geq n_{1}-1
$$

avec égalité dans les deux cas si et seulement si $G_{1}$ est l'étoile $S_{n_{1}}$.

Ainsi, les branches d'un graphe unicyclique minimisant la somme des distances (et par conséquent la distance moyenne), sont des étoiles.

Soit, maintenant $G$ un graphe unicyclique contenant au moins deux branches et dont toutes les branches sont des étoiles. Soit $W$ l'ensemble des sommets pendants de $G$. Pour chaque sommet de $v \in W$, notons la somme des distances de $v$ vers les sommets de $C$

$$
\sigma(v, C)=\sum_{x \in C} d(v, x)
$$

Alors

$$
\begin{aligned}
\sigma(G) & =\sigma(C)+\sum_{v \in V \backslash C} \sigma(v, C)+\frac{1}{2} \sum_{x, y \in V \backslash C} d(x, y) \\
& =\sigma(C)+(n-g) \sigma(v, C)+\frac{1}{2} \sum_{x, y \in V \backslash C} d(x, y) \\
& \geq \sigma(C)+(n-g) \sigma(v, C)+(n-g)(n-g-1) .
\end{aligned}
$$

Cette dernière borne est atteinte si et seulement si $d(x, y)=2$ pour tout $x, y \in$ $V \backslash C$; ce qui correspond à un graphe unicyclique avec une seule branche (qui est une étoile).

Pour avoir la formule de la borne, il suffit d'appliquer la Lemme 2.1 au navet $T_{n, g}$ avec $G_{1} \equiv C_{g}$ et $G_{2} \equiv S_{n-g+1}$.

Proposition 2.4. Si $g$ est fixé et $(2 n+3) / 3 \leq g \leq n-1$, alors $G$ est un graphe unicyclique, et par conséquent

$$
\bar{l} \geq\left\{\begin{array}{cl}
\frac{2}{g}-\frac{g^{2}-2 n g+8(n-1)}{4 n(n-1)} & \text { si g est pair } \\
\frac{2}{g}-\frac{g^{3}-2 n g^{2}+(8 n-9) g+2 n}{4 g n(n-1)} & \text { si g est impair }
\end{array}\right.
$$

avec égalité si et seulement si $G$ est le navet $T_{n, g}$.

Démonstration. Le fait que $G$ soit un graphe unicyclique, sous la condition de la proposition, est une conséquence immédiate de [13], Theorem 2. Le reste suit de la Proposition 2.3 ci-dessus. 
Proposition 2.5. Si $g=5$ ou $g=6$, alors

$$
\frac{\bar{l}}{g} \geq \frac{3}{10}
$$

avec égalité si et seulement si $G$ est $C_{5}$ ou $C_{6}$.

Démonstration. Il est facile de voir que l'égalité est vraie pour $C_{5}$ et $C_{6}$.

Si $g=5$, alors le diamètre $D$ de $G$ vérifie $D \geq 2$. Par conséquent

$$
\sigma(G) \geq m+2\left(\frac{n(n-1)}{2}-m\right)=n(n-1)-m
$$

où $m$ désigne la taille de $G$. Ainsi

$$
\bar{l}=\frac{2 \sigma(G)}{n(n-1)} \geq 2-\frac{2 m}{n(n-1)} .
$$

Pour que $\bar{l} / g>3 / 10$, i.e., $\bar{l}>3 / 2$, il suffit que $m<n(n-1) / 4$. Or d'après [13], $m \leq$ $n \sqrt{n-1} / 2$ dans tout graphe de maille $g=5$. Puisque $n \sqrt{n-1} / 2<n(n-1) / 4$ pour tout $n>5$, l'inégalité stricte est démontrée.

Si $g=6$ et $n \geq 7$, alors $D \geq 3$ et $G$ contient au moins 5 paires de sommets à distance 3 . Par conséquent

$$
\sigma(G) \geq m+2\left(\frac{n(n-1)}{2}-m-5\right)+15=n(n-1)-m+5
$$

et

$$
\bar{l} \geq 2-\frac{2 m}{n(n-1)}+\frac{10}{n(n-1)} .
$$

Pour que $\bar{l} / g>3 / 10$, i.e., $\bar{l}>9 / 5$, il suffit que $m<n(n-1) / 10+5$. Or d'après [12], $m \leq n(1+\sqrt{2 n-3}) / 4$ dans tout graphe de maille $g=6$. Il est facile de vérifier que

$$
\frac{n(1+\sqrt{2 n-3})}{4}<\frac{n(n-1)}{10}+5
$$

pour tout $n \geq 7$. Ainsi, l'inégalité stricte est démontrée.

\section{L'INDEX}

Dans cette section, nous démontrons deux conjectures structurelles sur les graphes minimisant la somme et le produit de la maille et de l'index. 
Proposition 3.1 (Conjecture 3 de [4]). Sur l'ensemble de tous les graphes connexes d'ordre $n \geq 4$, de maille finie $g$ et d'index $\lambda_{1}, g+\lambda_{1}$ est minimum pour le monocle $S_{3, n-3}$. De plus pour tout $t>0$, il existe $n_{t}$ tel que pour tout $n \geq n_{t}$, $3+\sqrt{5}-t<g\left(S_{3, n-3}\right)+\lambda_{1}\left(S_{3, n-3}\right)<3+\sqrt{5}$.

Démonstration. D'abord, il est à noter que l'index est décroissant par rapport à la supression d'arêtes dans un graphe [9]. Donc, il suffit de démontrer le résultat pour les graphes unicycliques.

D'une part, vu la relation $\lambda_{1} \leq \Delta$ (avec égalité si et seulement si le graphe est régulier) [9], on a $g\left(S_{3, n-3}\right)+\lambda_{1}\left(S_{3, n-3}\right)<6$. D'autre part, vu la relation $\lambda_{1} \geq \bar{d}=2 m / n$ [9], on a $g(G)+\lambda_{1}(G) \geq 6$, pour tout graphe unicyclique de maille $g(G) \geq 4$. Ainsi, on peut se limiter à l'ensemble des graphes unicyclique de maille $g=3$. Alors, il suffit de démontrer que $\lambda_{1}(G) \geq \lambda_{1}\left(S_{3, n-3}\right)$, pour tout graphe unicyclique de maille $g=3$. Ceci est prouvé dans [10] comme corollaire d'un résultat plus fort de [15].

Soit $G$ un graphe et $u$ un sommet de $G$. Notons $H$ le sous-graphe de $G$ issu de la suppression de $u$. Soit $G_{n}$ le graphe obtenu de $G$ et $P_{n}$ en reliant une extrémité de $P_{n}$ à $u$. Notons $r_{n}$ l'index du graphe $G_{n}, c-\grave{a}-d, r_{n}=\lambda_{1}\left(G_{n}\right)$. D'une part, comme l'ajout d'une arête augmente (strictement) l'index, alors $r_{n+1}>r_{n}$. D'autre part, dans [14] (voir aussi [9], p. 78), il est démontré que $\lim _{n \rightarrow \infty} r_{n}=r$ où $r$ est la plus grande solution de

$$
\frac{1}{2}\left(\lambda+\sqrt{\lambda^{2}-4}\right) P_{G}(\lambda)-P_{H}(\lambda)=0
$$

où $P_{G}$ et $P_{H}$ sont les polynômes caractéristiques de $G$ et $H$ respectivement. Dans notre cas, on prend $G \equiv K_{3}$ et $H \equiv P_{2}$, et ainsi $S_{3, n-3} \equiv G_{n-3}$, avec $P_{G}(\lambda)=$ $(\lambda-2)(\lambda+1)^{2}$ et $P_{H}(\lambda)=\lambda^{2}-1$. Donc (3.1) devient

$$
\frac{1}{2}\left(\lambda+\sqrt{\lambda^{2}-4}\right)(\lambda-2)(\lambda+1)^{2}-\left(\lambda^{2}-1\right)=0
$$

dont la plus grande solution est $r=\sqrt{5}$ (qui s'obtient en utilisant le logiciel Maple par exemple). Ainsi, la deuxième partie de la proposition s'ensuit.

Proposition 3.2 (Conjecture 4 de [4]). Sur l'ensemble de tous les graphes connexes d'ordre $n \geq 4$, de maille finie $g$ et d'index $\lambda_{1}$, le produit $g \cdot \lambda_{1}$ est minimum pour le monocle $S_{3, n-3}$. De plus pour tout $t>0$, il existe $n_{t}$ tel que pour tout $n \geq n_{t}, 3 \sqrt{5}-t<g\left(S_{3, n-3}\right) \cdot \lambda_{1}\left(S_{3, n-3}\right)<3 \sqrt{5}$.

Démonstration. Comme dans le cas de la Proposition 3.1, il suffit de démontrer le résultat pour les graphes unicycliques. Pour un graphe unicyclique de maille finie $g \geq 4$, on a $g \cdot \lambda_{1} \geq 8 m / n=8$ alors que le monocle $S_{3, n-3}$ de maille 3 
vérifie $g\left(S_{3, n-3}\right) \cdot \lambda_{1}\left(S_{3, n-3}\right)=3 r_{n-3}<3 r=3 \sqrt{5}<8$. Donc $g \lambda_{1}$ est minimum pour un graphe de maille 3 et un raisonnement analogue à celui de la preuve de la Proposition 3.1 permet de conclure.

\section{L'INDICE DE RANDIĆ}

Les conjectures 6 et 8 de [4], c-à-d, $R-g \geq n / 2$ et $R / g \geq 1 / 2$ sont démontrées dans [5].

Dans cette section, nous démontrons les bornes inférieures sur $R+g$ et $R \cdot g$. Notons qu'une preuve partielle de ces bornes, valable pour les graphes unicycliques, a été très récemment donnée dans [16]. En premier lieu, nous démontrons deux résultats intermédiaires qui donnent des bornes inférieures sur l'indice de Randić.

Lemme 4.1. Soit $G=(V, E)$ un graphe connexe et uv une arête pendante de $G$ avec $d(u)=1$ et $d=d(v) \geq 3$. Si au moins deux voisins de $v$ ont un degré au moins 2, alors

$$
\begin{aligned}
R(G)-R(G-u) & \geq \sqrt{d}-\sqrt{d-1}+\frac{2-\sqrt{2}}{\sqrt{d-1}}-\frac{2-\sqrt{2}}{\sqrt{d}} \\
& \geq \sqrt{n-1}-\sqrt{n-2}+\frac{2-\sqrt{2}}{\sqrt{n-2}}-\frac{2-\sqrt{2}}{\sqrt{n-1}}
\end{aligned}
$$

avec égalité si et seulement si $G$ est $T_{n, 3}$.

Démonstration. Soit $u v \in E$ une arête telle que $d(u)=1, d(v)=d \geq 3$. Notons $N(v)$ l'ensemble des voisins de $v$ et considérons $w_{1}, w_{2} \in N(v)$ tels que $d\left(w_{1}\right), d\left(w_{2}\right) \geq 2$. Soit $S_{v}$ la somme des poids des arêtes, autres que $u v$, incidentes au sommet $v$. Noter que

$$
R(G)-R(G-u)=\frac{1}{\sqrt{d}}+S_{v}-S_{v} \sqrt{\frac{d}{d-1}}
$$

On a

$$
\begin{aligned}
S_{v} & =\sum_{x \in N(v) \backslash\{u\}} \frac{1}{\sqrt{d \cdot d(x)}} \\
& =\frac{1}{\sqrt{d \cdot d\left(w_{1}\right)}}+\frac{1}{\sqrt{d \cdot d\left(w_{2}\right)}}+\sum_{x \in N(v) \backslash\left\{u, w_{1}, w_{2}\right\}} \frac{1}{\sqrt{d \cdot d(x)}} \\
& \leq \frac{2}{\sqrt{2 d}}+\frac{d-3}{\sqrt{d}} .
\end{aligned}
$$


Alors

$$
\begin{aligned}
R(G)-R(G-u) & \geq \frac{1}{\sqrt{d}}+\left(\frac{2}{\sqrt{2 d}}+\frac{d-3}{\sqrt{d}}\right)\left(1-\sqrt{\frac{d}{d-1}}\right) \\
& =\frac{1}{\sqrt{d}}+\left(\frac{d-3+\sqrt{2}}{\sqrt{d}}\right)\left(1-\frac{\sqrt{d}}{\sqrt{d-1}}\right) \\
& =\frac{d-2+\sqrt{2}}{\sqrt{d}}-\sqrt{d-1}+\frac{2-\sqrt{2}}{\sqrt{d-1}} \\
& =\sqrt{d}-\sqrt{d-1}+\frac{2-\sqrt{2}}{\sqrt{d-1}}-\frac{2-\sqrt{2}}{\sqrt{d}} .
\end{aligned}
$$

Il est facile de voir que cette dernière borne n'est atteinte que si $d=n-1$ et $v$ admet exactement deux voisins de degré 2 . Ainsi, le graphe correspondant à l'égalité est le navet $T_{n, 3}$.

Lemme 4.2. Soit $G=(V, E)$ un graphe connexe et uv une arête pendante de $G$ avec $d(u)=1$ et $d=d(v) \geq 2$. Si au moins un voisin de $v$ est de degré au moins 2, alors

$$
R(G)-R(G-u) \quad \geq \quad\left(\frac{d-1}{\sqrt{d}}+\frac{1}{\sqrt{2 d}}\right)-\left(\frac{d-2}{\sqrt{d-1}}+\frac{1}{\sqrt{2(d-1)}}\right) .
$$

de plus cette borne est une fonction décroissante en $d$.

Démonstration. Soit $u v \in E$ une arête telle que $d(u)=1, d(v)=d \geq 2$. Considérons $w \in N(v)$ tel que $d(w) \geq 2$. Soit $S_{v}$ la somme des poids des arêtes, autre que $u v$, incidentes au sommet $v$. Notons que

$$
R(G)-R(G-u)=\frac{1}{\sqrt{d}}+S_{v}-S_{v} \sqrt{\frac{d}{d-1}}
$$

On a

$$
\begin{aligned}
S_{v} & =\sum_{x \in N(v) \backslash\{u\}} \frac{1}{\sqrt{d \cdot d(x)}} \\
& =\frac{1}{\sqrt{d \cdot d(w)}}+\sum_{x \in N(v) \backslash\{u, w\}} \frac{1}{\sqrt{d \cdot d(x)}} \\
& \leq \frac{1}{\sqrt{2 d}}+\frac{d-2}{\sqrt{d}} .
\end{aligned}
$$


Alors

$$
\begin{aligned}
R(G)-R(G-u) & \geq \frac{1}{\sqrt{d}}+\left(\frac{1}{\sqrt{2 d}}+\frac{d-2}{\sqrt{d}}\right)\left(1-\sqrt{\frac{d}{d-1}}\right) \\
& =\frac{1}{\sqrt{d}}+\left(\frac{d-2+\frac{1}{\sqrt{2}}}{\sqrt{d}}\right)\left(1-\frac{\sqrt{d}}{\sqrt{d-1}}\right) \\
& =\frac{1}{\sqrt{d}}+\frac{d-2+\frac{1}{\sqrt{2}}}{\sqrt{d}}-\frac{d-2+\frac{1}{\sqrt{2}}}{\sqrt{d-1}} \\
& =\left(\frac{d-1}{\sqrt{d}}+\frac{1}{\sqrt{2 d}}\right)-\left(\frac{d-2}{\sqrt{d-1}}+\frac{1}{\sqrt{2(d-1)}}\right) .
\end{aligned}
$$

Ceci complète la preuve de la borne. En utilisant la dérivée de la borne, considérée comme une fonction en $d$, il est facile de voir qu'elle est décroissante.

Théorème 4.1 (Conjectures 7 et 9 de [4]). Soit $G=(V, E)$ un graphe connexe sur $n \geq 3$ sommets d'indice de Randić $R$ et de maille finie $g$. Alors

$$
R+g \geq \frac{n-3+\sqrt{2}}{\sqrt{n-1}}+\frac{7}{2} \quad \text { and } \quad R \cdot g \geq \frac{3 n-9+3 \sqrt{2}}{\sqrt{n-1}}+\frac{3}{2}
$$

avec égalité si et seulement si $G$ est $T_{n, 3}$.

Notons que l'indice de Randić de $T_{n, 3}$ est donné par

$$
R\left(T_{n, 3}\right)=\frac{n-3+\sqrt{2}}{\sqrt{n-1}}+\frac{1}{2}
$$

Avant de démontrer le théorème, nous donnons deux résultats intermédiaires.

Lemme 4.3. Si la maille d'un graphe $H$ vaut au moins 4, alors

$$
R+g>\frac{n-3+\sqrt{2}}{\sqrt{n-1}}+\frac{7}{2} \quad \text { et } \quad R \cdot g>\frac{3 n-9+3 \sqrt{2}}{\sqrt{n-1}}+\frac{3}{2} .
$$

Démonstration. Il est bien connu [6] que pour tout graphe $R \geq \sqrt{n-1}$, avec égalité uniquement pour $S_{n}$. Donc si $g \geq 4$, alors

$R+g>\sqrt{n-1}+4>\frac{n-3+\sqrt{2}}{\sqrt{n-1}}+\frac{7}{2} \quad$ et $R \cdot g>4 \sqrt{n-1}>\frac{3 n-9+3 \sqrt{2}}{\sqrt{n-1}}+\frac{3}{2}$.

Ainsi, les minima sur $R+g$ et sur $R \cdot g$ sont atteints pour des graphes de maille $g=3$.

Lemme 4.4. Si le degré minimum d'un graphe connexe $H$ d'ordre $n \geq 4$ vaut au moins 2, alors

$$
R(H) \geq R\left(T_{n, 3}\right) .
$$


Démonstration. Dans [11], il a été prouvé que si $\delta \geq 2$ alors

$$
R \geq \sqrt{2(n-1)}+\frac{1}{n-1}-\frac{\sqrt{2}}{\sqrt{n-1}} .
$$

Cette borne est plus grande que l'indice de Randić de $T_{n, 3}$ pour tout $n \geq 4$.

Preuve du Théorème 4.1. La preuve est par récurrence sur le nombre de sommets $n$.

Si $n=3$, il y a un seul graphe de maille finie qui $K_{3} \equiv T_{3,3}$. Le résultat est vrai dans ce cas.

Pour $n \geq 4$, d'après les Lemmes 4.3 et 4.4 , on peut supposer que $\delta(G)=1$ et $g(G)=3$.

Si $n=4$, il y a un seul graphe avec un degré minimum $\delta=1$ et une maille $g=3$, qui est $T_{4,3}$. Donc le résultat est vrai pour $n=4$.

Supposons le résultat vrai pour tout graphe sur moins de $n$ sommets avec $n \geq 5$. Soit $G$ un graphe connexe sur $n$ sommets avec $\delta(G)=1$ et $g(G)=3$. Soit $u$ un sommet de $G$ de degré 1 . Son unique voisin $v$ possède au moins un voisin de degré au moins 2 puisque $G-u$ n'est pas une étoile.

- Si $v$ possède au moins deux voisins de degré au moins 2 , alors en utilisant le Lemma 4.1,

$$
R(G) \geq R(G-u)+\sqrt{n-1}-\sqrt{n-2}+\frac{2-\sqrt{2}}{\sqrt{n-2}}-\frac{2-\sqrt{2}}{\sqrt{n-1}} .
$$

Par hypothèse de récurrence

$$
R(G-u) \geq \frac{n-4+\sqrt{2}}{\sqrt{n-2}}+\frac{1}{2} .
$$

Donc

$$
\begin{aligned}
R(G) & \geq \frac{n-4+\sqrt{2}}{\sqrt{n-2}}+\frac{1}{2}+\sqrt{n-1}-\sqrt{n-2}+\frac{2-\sqrt{2}}{\sqrt{n-2}}-\frac{2-\sqrt{2}}{\sqrt{n-1}} \\
& =\left(\frac{n-4+\sqrt{2}}{\sqrt{n-2}}-\sqrt{n-2}+\frac{2-\sqrt{2}}{\sqrt{n-2}}\right)+\left(\sqrt{n-1}-\frac{2-\sqrt{2}}{\sqrt{n-1}}\right)+\frac{1}{2} \\
& =\frac{n-3+\sqrt{2}}{\sqrt{n-1}}+\frac{1}{2}=R\left(T_{n, 3}\right) .
\end{aligned}
$$

L'égalité est vraie si et seulement si $G$ est $T_{n, 3}$. Ainsi, dans ce cas, le résultat est vrai. 
- Si $v$ possède exactement un voisin de degré au moins 2 , alors le sommet $v$ est adjacent à au plus un sommet d'un triangle de $G$. Alors $d \leq n-3$ et $n \geq 5$. En utilisant le Lemme 4.2,

$$
\begin{aligned}
R(G) & \geq R(G-u)+\left(\frac{d-1}{\sqrt{d}}+\frac{1}{\sqrt{2 d}}\right)-\left(\frac{d-2}{\sqrt{d-1}}+\frac{1}{\sqrt{2(d-1)}}\right) \\
& \geq R(G-u)+\left(\frac{n-4}{\sqrt{n-3}}+\frac{1}{\sqrt{2(n-3)}}\right)-\left(\frac{n-5}{\sqrt{n-4}}+\frac{1}{\sqrt{2(n-4)}}\right) .
\end{aligned}
$$

Par hypothèse de récurrence

$$
\begin{aligned}
R(G) \geq & R\left(T_{n-1,3}\right)+\left(\frac{n-4}{\sqrt{n-3}}+\frac{1}{\sqrt{2(n-3)}}\right)-\left(\frac{n-5}{\sqrt{n-4}}+\frac{1}{\sqrt{2(n-4)}}\right) \\
= & \frac{n-4+\sqrt{2}}{\sqrt{n-2}}+\frac{1}{2}+\left(\frac{n-4}{\sqrt{n-3}}+\frac{1}{\sqrt{2(n-3)}}\right)-\left(\frac{n-5}{\sqrt{n-4}}+\frac{1}{\sqrt{2(n-4)}}\right) \\
= & R\left(T_{n, 3}\right)-\frac{n-3+\sqrt{2}}{\sqrt{n-1}}+\frac{n-4+\sqrt{2}}{\sqrt{n-2}} \\
& +\left(\frac{n-4}{\sqrt{n-3}}+\frac{1}{\sqrt{2(n-3)}}\right)-\left(\frac{n-5}{\sqrt{n-4}}+\frac{1}{\sqrt{2(n-4)}}\right) \\
= & R\left(T_{n, 3)-(} \frac{n-3}{\left.\sqrt{n-1}+\frac{2}{\sqrt{2(n-1)}}\right)+\left(\frac{n-4}{\sqrt{n-2}}+\frac{1}{\sqrt{2(n-2)}}\right)}\right. \\
& +\left(\frac{n-4}{\sqrt{n-3}}+\frac{1}{\sqrt{2(n-3)}}\right)-\left(\frac{n-5}{\sqrt{n-4}}+\frac{1}{\sqrt{2(n-4)}}\right) \\
= & R\left(T_{n, 3}\right)+\left(1-\frac{1}{\sqrt{2}}\right)\left(\frac{1}{\sqrt{n-1}}-\frac{1}{\sqrt{n-2}}+\frac{1}{\sqrt{n-4}}-\frac{1}{\sqrt{n-3}}\right) \\
& +(\sqrt{n-2}+\sqrt{n-3}-\sqrt{n-1}-\sqrt{n-4}) \\
& -\left(1-\frac{1}{\sqrt{2}}\right)\left(\frac{1}{\sqrt{n-2}}-\frac{1}{\sqrt{n-1}}\right) \cdot \\
& (1)
\end{aligned}
$$

Posons

$$
a_{n}=\frac{1}{\sqrt{n-1}}-\frac{1}{\sqrt{n-2}}+\frac{1}{\sqrt{n-4}}-\frac{1}{\sqrt{n-3}}
$$

et

$$
b_{n}=\sqrt{n-2}+\sqrt{n-3}-\sqrt{n-1}-\sqrt{n-4}-\left(1-\frac{1}{\sqrt{2}}\right)\left(\frac{1}{\sqrt{n-2}}-\frac{1}{\sqrt{n-1}}\right) .
$$

Pour conclure, il suffit de démontrer que $a_{n}>0$ et $b_{n}>0$ pour tout $n \geq 5$. 
Considérons $a_{n}$ en premier.

$$
\begin{aligned}
a_{n} & =\frac{\sqrt{n-2}-\sqrt{n-1}}{\sqrt{(n-1)(n-2)}}+\frac{\sqrt{n-3}-\sqrt{n-4}}{\sqrt{(n-3)(n-4)}} \\
& =\frac{-1}{(\sqrt{n-2}+\sqrt{n-1}) \sqrt{(n-1)(n-2)}}+\frac{1}{(\sqrt{n-3}+\sqrt{n-4}) \sqrt{(n-3)(n-4)}} \\
& >0 .
\end{aligned}
$$

Maintenant considérons $b_{n}$.

$$
\begin{aligned}
b_{n}= & \frac{-1}{\sqrt{n-1}+\sqrt{n-2}}+\frac{1}{\sqrt{n-3}+\sqrt{n-4}} \\
& -\left(1-\frac{1}{\sqrt{2}}\right) \cdot \frac{1}{(\sqrt{n-1}+\sqrt{n-2}) \sqrt{(n-1)(n-2)}} \\
= & \frac{1}{\sqrt{n-3}+\sqrt{n-4}}-\frac{1}{\sqrt{n-1}+\sqrt{n-2}} \cdot\left(1+\frac{1}{\sqrt{(n-1)(n-2)}}\right) .
\end{aligned}
$$

D'où $b_{n}>0$ si et seulement si

$$
\begin{gathered}
\frac{1}{\sqrt{n-3}+\sqrt{n-4}}>\frac{1}{\sqrt{n-1}+\sqrt{n-2}} \cdot\left(1+\frac{1-\frac{1}{\sqrt{2}}}{\sqrt{(n-1)(n-2)}}\right) \\
\Leftrightarrow \quad \frac{1}{\sqrt{n-3}+\sqrt{n-4}}>\frac{1}{\sqrt{n-1}+\sqrt{n-2}} \cdot \frac{\sqrt{(n-1)(n-2)}+\frac{\sqrt{2}-1}{\sqrt{2}}}{\sqrt{(n-1)(n-2)}} \\
\Leftrightarrow \quad \sqrt{n-3}+\sqrt{n-4}<(\sqrt{n-1}+\sqrt{n-2}) \cdot \frac{\sqrt{(n-1)(n-2)}}{\sqrt{(n-1)(n-2)}+\frac{\sqrt{2}-1}{\sqrt{2}}} \\
\Leftrightarrow \quad \sqrt{n-3}+\sqrt{n-4}<(\sqrt{n-1}+\sqrt{n-2}) \cdot\left(1-\frac{\sqrt{2}-1}{\sqrt{2}}\right.
\end{gathered}
$$

Prenons le membre de gauche,

$$
\sqrt{n-3}+\sqrt{n-4}<2 \sqrt{n-3}
$$

Maintenant, prenons le membre de droite,

$$
(\sqrt{n-1}+\sqrt{n-2}) \cdot\left(1-\frac{\frac{\sqrt{2}-1}{\sqrt{2}}}{\sqrt{(n-1)(n-2)}+\frac{\sqrt{2}-1}{\sqrt{2}}}\right)>2 \sqrt{n-2} \cdot\left(1-\frac{\frac{\sqrt{2}-1}{\sqrt{2}}}{n-2}\right) .
$$


Ainsi pour prouver que $b_{n}>0$, il suffit de prouver que

$$
\sqrt{n-3}<\sqrt{n-2} \cdot\left(1-\frac{\frac{\sqrt{2}-1}{\sqrt{2}}}{n-2}\right)
$$

En prenant le carré des deux membres de cette dernière inégalité, on doit prouver que

$$
n-3<(n-2) \cdot\left(1-\frac{2-\sqrt{2}}{n-2}+\frac{\frac{(\sqrt{2}-1)^{2}}{2}}{(n-2)^{2}}\right)=n-3+(\sqrt{2}-1)+\frac{\frac{(\sqrt{2}-1)^{2}}{2}}{(n-2)},
$$

qui est évidemment vrai. Ceci complète la preuve.

\section{LE NOMBRE DE DOMINATION}

Dans cette section, nous démontrons les bornes inférieures sur $\beta-g$ et $\beta / g$.

Proposition 5.1 (Conjectures 10 et 11 de [4]). Soit $G=(V, E)$ un graphe connexe sur $n \geq 3$ sommets de nombre de domination $\beta$ et de maille finie $g$. Alors

$$
\beta-g \geq-\left\lfloor\frac{2 n}{3}\right\rfloor \quad \text { and } \quad \frac{\beta}{g} \geq \frac{1}{3} .
$$

La borne est atteinte pour tout valeur de $n \geq 3$.

Démonstration. Soit $C$ un cycle de longueur $g$ de $G$. Un ensemble dominant de $G$ doit contenir au moins $\lceil g / 3\rceil$ sommets pour dominer le cycle $C$. Donc

$$
\beta-g \geq\left\lceil\frac{g}{3}\right\rceil-g=-\left\lfloor\frac{2 g}{3}\right\rfloor \geq-\left\lfloor\frac{2 n}{3}\right\rfloor \quad \text { and } \quad \frac{\beta}{g} \geq \frac{\left\lceil\frac{g}{3}\right\rceil}{g} \geq \frac{1}{3} .
$$

Ainsi la borne est prouvée.

Il est facile de vérifier que la borne inférieure sur la différence est atteinte pour les cycles (entre autre), et que la borne sur le rapport est atteinte pour tout graphe de degré maximum $\Delta=n-1$ et contenant au moins $n$ arêtes.

En conclusion les résultats prouvés dans cet article confirment les conclusions de [2] : (i) les conjectures obtenues par AGX 2 sont très souvent vraies; (ii) elles vont de cas très faciles tels que la Proposition 5.1 à des cas plus complexes tels que les Théorèmes 1 et 2, et demeurent parfois ouvertes telles que la Conjecture 1 modifiée. 


\section{RÉFÉRENCES}

[1] M. Aouchiche, Comparaison automatisée d'invariants en théorie des graphes. Ph.D. Thesis, École Polytechnique de Montréal, February (2006). Disponible sur www.gerad.ca/ a agx.

[2] M. Aouchiche, G. Caporossi and P. Hansen, Variable neighborhood search for extremal graphs. 20. Automated comparison of graph invariants. MATCH Commun. Math. Comput. Chem. 58 (2007) 365-384.

[3] M. Aouchiche, J.-M. Bonnefoy, A. Fidahoussen, G. Caporossi, P. Hansen, L. Hiesse, J. Lacheré and A. Monhait, Variable Neighborhood Search for Extremal Graphs. 14. The AutoGraphiX 2 System, in Global Optimization: From theory to implementation, edited by L. Liberti and N. Maculan, Springer (2006) 281-310.

[4] M. Aouchiche and P. Hansen, Recherche à voisinage variable de graphes extrémaux. XIII. À propos de la maille. (French) RAIRO-Oper. Res. 39 (2005) 275-293.

[5] M. Aouchiche, P. Hansen and M. Zheng, Variable neighborhood search for extremal graphs. 19. Further conjectures and results about the Randić index. MATCH Commun. Math. Comput. Chem. 58 (2007) 83-102.

[6] B. Bollobás and P. Erdös, Graphs of extremal weights. Ars Combin. 50 (1998) 225-233.

[7] J.A. Bondy and F.Y. Halberstam, Parity theorems for paths and cycles in graphs. J. Graph Theory 10 (1986) 107-115.

[8] G. Caporossi and P. Hansen, Variable neighborhood search for extremal graphs. I. The AutGraphiX system. Disc. Math. 212 (2000) 29-44.

[9] D. Cvetković, M. Doob and H. Sachs, Spectra of graphs - Theory and application. Academic Press, New York (1982).

[10] D. Cvetković and P. Rowlinson, Spectra of unicyclic graphs. Graphs and combinatorics 3 (1987) $7-23$.

[11] C. Delorme, O. Favaron and D. Rautenbach, On the Randić index. Discrete Math. 257 (2002) 29-38.

[12] F.M. Dong and K.M. Koh, The Sizes of Graph with Small Girth. Bull. Inst. Combin. Appl. 18 (1996) 33-44.

[13] R.D. Dutton and R.C. Brigham, Edges in Graphs with Large Girth. Graphs Combin. 7 (1991) 315-321.

[14] A.J. Hoffman, On Limit Points of Spectral Radii of Non-Negative Symmetric Integral Matrices, in Graph Theory and Applications. Lect. Notes Math. 303, edited by Y. Alavi, D.R. Lick, A.T. White, Springer-Verlag, Berlin (1972) 165-172.

[15] Q. Li, and K.Q. Feng, On the largest eigenvalue of a graph. Acta Math. Appl. Sinica 2 (1979) $167-175$.

[16] G. Liu, Y. Zhu and J. Cai, On the Randić index of unicyclic graphs with girth $g$. MATCH Commun. Math. Comput. Chem. 58 (2007) 127-138.

[17] P. Turán, An extremal problem in graph theory. Mat. Fiz. Lapok 48 (1941) 436-452. 